\title{
Naturalización de los contenidos y objetivos de apren- dizaje: acerca del énfasis que los futuros maestros de primaria ponen en los aspectos técnico-pedagógicos
}

\section{Naturalization of content-matter and learning objectives: about the emphasis that future primary school teachers to grant to the pedagogical technical aspects}

\author{
Héctor CÁRCAMO VÁSQUEZ \\ Universidad del Bío-Bío \\ hcarcamo@ubiobio.cl \\ Proyecto Mecesup-UBB 0704. Proyecto de Investigación DIUBB 142424 2/RS
}

Recibido: 16 de mayo de 2014

Aceptado: 25 de noviembre de 2014

\begin{abstract}
Resumen
El objetivo de este artículo es reconocer las condiciones que propician la naturalización de los contenidos a enseñar por parte de los futuros profesores de educación primaria. La metodología empleada corresponde a la cualitativa con enfoque etnográfico. Para la recogida de datos se utilizó la revisión documental y la entrevista en profundidad. La investigación se realizó en la ciudad de Madrid, específicamente el área de estudio estuvo conformada por dos universidades públicas y dos universidades privadas. La investigación se inició el año 2010 y finalizó a mediados de 2013. Los resultados obtenidos nos permiten plantear que lo que prima en el contexto de la formación inicial del profesorado de educación primaria y, muy probablemente, durante los primeros años de la carrera docente es una lógica instrumental cuyo reflejo más claro se encuentra en la hegemonía de una 'tecnología instruccional' dentro de los espacios escolares, sean del nivel que sean.
\end{abstract}

Palabras clave: etnografía de la educación; educación universitaria; pedagogía; rol docente.

\begin{abstract}
The aim of this paper is to recognize the conditions conducive to the naturalization of content-matter by future primary school teachers. The research was carried out using a qualitative methodology with an ethnographic approach. The main techniques used were interviews, as well as a review of documentation. The study was conducted in the city of Madrid in two public and two private universities. Research begun in 2010 and finished in mid-2013. The results enable us to state that what prevails in the context of initial teacher training and, most likely, during the early years of the teaching career is an instrumental logic of which the clearest reflection is the hegemony of 'instructional technology' within the school spaces, whatever level they are.
\end{abstract}

Keywords: ethnography of education; university education; pedagogy; teaching role. 
SUMARIO: 1. Introducción: consideraciones generales. 2. Genealogía del discurso pedagógico constructivista en España. 3. Método y técnicas. 4. Presentación de resultados. 4.1. Acerca del discurso institucional y su influencia en las imágenes de los estudiantes de magisterio de primaria. 4.2. Por qué el énfasis en el cómo: una aproximación desde el discurso de los estudiantes. 5. A modo de conclusión. La práctica y lo práctico en la enseñanza: una síntesis como consecuencia de la tecnología instruccional. 6. Referencias bibliográficas.

Referencia normalizada: Cárcamo Vásquez, H. (2015) Naturalización de los contenidos y objetivos de aprendizaje: acerca del énfasis que los futuros maestros de primaria ponen en los aspectos técnico-pedagógicos, en Revista de Antropología Social 24, 271-285.

\section{Introducción: consideraciones generales}

Para iniciar diremos que la naturalización la entendemos como el proceso que se da cuando las propiedades de un fenómeno social son separadas de la red de relaciones de las que participa, y se las reconoce erróneamente como perteneciéndoles al fenómeno en sí mismo. Dicho esto, cabe señalar que la pregunta que orienta el desarrollo de este trabajo es: ¿qué condiciones propician la naturalización de los contenidos que llevan a restringir el rol de maestro al ámbito de la praxis pedagógica? Sobre ello se pueden barajar algunas hipótesis: 1) los futuros maestros asumen como algo natural que lo que deben hacer es enseñar, como algo que no se cuestiona, debido al peso que tiene la didáctica (y las Didácticas en cuanto asignaturas) en su formación inicial docente; 2) la falta de una experiencia continuada en un aula de primaria y la de participar en la elaboración de un proyecto educativo de centro como parte de un equipo docente dificultan a los estudiantes de magisterio adoptar una posición respecto a los contenidos a desarrollar en dicho espacio; y 3) los futuros profesores no se cuestionan los contenidos a enseñar por cuanto consideran que las didácticas pueden transformarse en dispositivos que contribuyan a un buen desempeño profesional.

Dado que lo que se visualiza al analizar los discursos de los futuros profesores de primaria es la presencia de un discurso pedagógico de carácter institucional, creemos pertinente detenernos en lo que hemos denominado genealogía del discurso pedagógico constructivista en España, para luego dar paso a la metodología utilizada en esta investigación, la presentación de resultados y las conclusiones del estudio.

\section{Genealogía del discurso pedagógico constructivista en España}

Para reflexionar sobre el discurso pedagógico institucional que se configura a partir de 1990 en España, es necesario referirse a la propuesta de reforma planteada en la Ley Orgánica de Ordenación General del Sistema Educativo (LOGSE), pues diversos autores (Marchesi, 2000; Marchesi y Martín, 1998; Martín y Coll, 2003; Pozo, 1996; Varela, 2007) coinciden en señalar que esta iniciativa de reforma supuso un cambio sustantivo del sistema educativo español; de hecho, las propuestas de reforma posteriores a ella han sido elaboradas sobre la base constructivista de la LOGSE, obviamente con matices de carácter político-ideológico que no pueden ignorarse. 
¿Por qué surge la necesidad de plantear una reforma en la década de 1980 en España?, ¿en qué aspectos se sostiene el debate para su formulación y puesta en marcha? Siguiendo los planteamientos de Marchesi y Martín (1998), un primer aspecto a tener en cuenta es la necesidad que se presenta a los profesores de repensar su quehacer, debido a los cambios sociales profundos que se estaban manifestando desde los años 80' como, por ejemplo, la internacionalización de la economía, la globalización de la comunicación y la información, el desarrollo científico y tecnológico, la desaparición de las barreras nacionales, el incremento del flujo migratorio, los cambios demográficos y familiares, la diversificación del empleo o el pluralismo ideológico y moral de la sociedad. Como consecuencia, durante los 90' la educación se vio enfrentada a un conjunto de retos, entre los que estaban la emergencia de una sociedad del conocimiento, la búsqueda de la calidad educativa, el objetivo aún inalcanzado de la igualdad educativa, así como el valor otorgado a la cultura de los centros y al aprendizaje en el aula.

Un segundo aspecto que permite entender por qué se necesitaba el cambio es la existencia de una gran desigualdad en el acceso a la educación; lo que se refleja en el hecho de que, por ejemplo, según Marchesi (2000: 50), el 22,7\% de la clase media-alta accediera a la formación universitaria, mientras que este porcentaje bajaba hasta sólo un $6,8 \%$ en el caso de la clase obrera. Por otro lado, la desigualdad en el acceso a la educación se veía profundizada en el aula debido a que el modelo de enseñanza-aprendizaje que se implementaba en ella atendía a la diversidad del alumnado, ya se derivara ésta de diferencias culturales y/o de necesidades educativas especiales (NEE), por medio de la segregación, una de cuyas manifestaciones se puede encontrar, por ejemplo, en la inexistencia de programas de integración de alumnos con NEE en aulas convencionales.

Aunque muchos son los argumentos que se esgrimen para justificar la reforma al sistema escolar en España (cambio en la estructura familiar, cambio en los valores, cambio en el mercado laboral, etc.,), al que se le otorga especial atención es a la desmotivación del alumnado. De acuerdo con Marchesi (2000), la constatación de la desmotivación del alumnado lleva a examinar los factores que pueden ayudar a comprender de manera más integral este fenómeno con el fin de revertirlo y alcanzar los objetivos escolares que se trazan desde la Administración Educativa. En tal sentido, comienza a indagarse acerca de los cambios que, concernientes al segmento de la población en edad escolar, están contribuyendo a esta desmotivación. Es así como se llega a establecer que estos cambios fueran principalmente tres: 1) cambio en las relaciones entre niños, jóvenes y adultos, que pasaron a ser más simétricas; 2) cambio alusivo al estilo educativo, pues empezó a deslegitimarse el estilo vertical de mandato-obediencia, reemplazándose por el de negociación y comunicación entre alumnos y maestros; 3) y, por último, consolidación de una cultura de aprendizaje que apela a lo audiovisual, pues la exposición magistral y la repetición de los contenidos de los libros de texto son cuestionadas en tanto que estrategias de enseñanza-aprendizaje.

Dado que la oportunidad de acceder a la educación obedece más bien a cuestiones de carácter macro-estructural, el profesorado no considera que pueda influir 
en ella. Por esto quizá tienda a centrar su atención en la necesidad de revertir la creciente desmotivación del alumnado, máxime cuando ésta se muestra como una realidad que los interpela día a día de manera directa en las interacciones con sus alumnos. Por otro lado, si se tiene en cuenta que la motivación ha sido un terreno fértil para la psicología, sobre todo en lo que se refiere a su conceptualización, teorización y abordaje metodológico, puede entenderse el protagonismo que la psicología ha adquirido como campo disciplinar que se ocupa (y se preocupa) de comprender las distintas etapas del desarrollo de la persona, así como también los procesos de aprendizaje que ésta realiza.

En psicología se han confrontado durante décadas dos enfoques diferentes respecto a los procesos de aprendizaje: el conductista y el constructivista. Los especialistas españoles en la materia, aunque no de forma unánime, fueron decantándose desde finales de los $80^{\prime}$ por el constructivismo, de modo que se fue configurando un discurso cada vez más hegemónico en torno a cuál era el enfoque más apropiado para dotar de eficacia al proceso de enseñanza-aprendizaje ${ }^{1}$ en la escuela y que, por tanto, fuera el más adecuado también para guiar las propuestas de reforma del sistema escolar español. De hecho, es la influencia de este enfoque en la LOGSE la que explica algunos de los grandes cambios que ésta presenta con respecto a las políticas educativas anteriores, pues se pasa de conceder una importancia exclusiva e irrestricta a los contenidos conceptuales —entendidos como ideas o imágenes mentales sobre las cosas (el saber) —, a una concepción más amplia de lo que son los contenidos educativos, que incorporan, además de los conceptos, los procedimientos, habilidades y estrategias (el saber hacer o contenidos procedimentales), así como también los valores, las actitudes y las normas (el saber ser, saber estar y saber comportarse o contenidos actitudinales) (Martín y Coll, 2003). Esto supone una transformación sustantiva de la manera en que el profesorado debe definir y poner en marcha la acción educativa, pues de prestar atención a qué enseñar (a los conocimientos que tienen que adquirirse) se pasa a cómo enseñar (a la praxis pedagógica que tiene que desarrollarse para que se generen capacidades).

Con todo, es preciso aclarar que el constructivismo, más que servir para definir pautas y estrategias de enseñanza-aprendizaje, lo que proporciona es un marco interpretativo para comprender lo que acontece en el interior de los sujetos cuando están implicados en procesos de conocimiento: por qué disciernen o no ciertas cosas o por qué distintos sujetos entienden cosas distintas frente a una misma situación. No obstante, este enfoque es adoptado y, mejor dicho, adaptado por el discurso institucional que circula en el Grado de Magisterio (/Maestro) de Educación Primaria, pues se considera útil para obtener un conjunto de dispositivos técnicos formalizados en las didácticas (como actividades y estrategias para el aprendizaje) que faciliten que el alumnado consiga los tan ansiados aprendizajes significativos. Conocer en qué consisten y saber usar este tipo de dispositivos es precisamente lo que demandan los futuros maestros cuando están cursando su formación inicial.

${ }^{1}$ La eficacia en este sentido está dirigida a incrementar la motivación del alumnado, así como también a lograr aprendizajes significativos entre los niños y los jóvenes en edad escolar. 
A diferencia del enfoque conductista, el enfoque constructivista se inclina hacia la delimitación de currículos más abiertos, pues proporcionan un conjunto de contenidos que operan más bien como orientaciones para la acción del maestro, por tanto, el profesorado tiene la posibilidad de asumir mayor protagonismo a la hora de seleccionar contenidos y no sólo actividades que restrinjan su acción al mero didactismo. ¿Cómo es, entonces, que se da entre los futuros profesores de educación primaria una naturalización de los contenidos, es decir, de lo que hay que enseñar? Una de las respuesta posibles a esta pregunta es que las universidades procuran proporcionar herramientas de carácter técnico-pedagógico, ya que éstas son mejor valoradas como competencias para el desempeño profesional. De hecho, como se ha dicho, los discursos de los futuros maestros de primaria también vehiculan una representación del desempeño profesional que lo hace depender del manejo de la técnica y de métodos de enseñanza, por considerar que los aspectos crítico-reflexivo, aunque importantes, no resuelven los actuales problemas de la educación y, en particular, la ya mencionada desmotivación del alumnado.

En consideración a lo expuesto en los párrafos precedentes, se torna necesario recordar cuál es el objetivo de este trabajo. A modo general diremos que lo que interesa, fundamentalmente, es reconocer las condiciones que propician la naturalización de los contenidos a enseñar por parte de los futuros profesores de educación primaria.

\section{Método y técnicas}

El diseño utilizado se sustentó en la metodología cualitativa, específicamente el enfoque etnográfico. La investigación se desarrolló en la ciudad de Madrid entre los años 2010 y 2013. El trabajo investigativo se realizó en dos universidades públicas y dos privadas; a todas hemos decidido cambiar sus nombres para asegurar la confidencialidad. Las públicas son la Universidad Los Robles y la Universidad Los Olmos; por su parte las privadas Universidad Los Cipreses, adscrita a la Universidad Los Robles, y la Universidad Las Acacias, adscrita a la Universidad Los Olmos.

Las técnicas de producción de información utilizadas corresponden a la revisión documental y las entrevistas en profundidad. En cuanto a la revisión documental, diremos que las fuentes principales de donde se obtuvo la información fue la documentación de carácter institucional de las universidades en las cuales centramos nuestra atención (se revisaron planes de estudio de la carrera de grado de educación primaria, guías docentes, prestándose atención a los contenidos establecidos, al tiempo destinado a ellos, así como también a la bibliografía declarada como obligatoria).

En lo que respecta a la técnica de entrevista, diremos que se trabajó a partir del establecimiento de bloques temáticos. Dado que los resultados que exponemos en este trabajo derivan de una investigación más amplia, aquí solo presentaremos los bloques temáticos directamente vinculados con la pregunta guía aquí planteada. Estos son los que se detallan a continuación: 1) Aspectos relativos al ingreso a la carrera. 2) Aspectos relativos al proceso de formación inicial docente. 3) Aspectos relativos al concepto de educación. Respecto de las técnicas empleadas, es 
relevante señalar que asumimos que la no utilización de la técnica de observación de prácticas sociales en contexto representa una debilidad del presente trabajo, pero a la vez deja abierta la posibilidad de continuar la línea de investigación con el fin de contrastar los elementos pesquisados a través de las técnicas de entrevistas etnográficas y la revisión documental con información producida desde la observación. Asimismo, indicar que el material empírico obtenido aporta los elementos suficientes para el desarrollo argumentativo y el cumplimiento del objetivo trazado.

En cuanto a los sujetos entrevistados fueron un total de 32 estudiantes, distribuidos equitativamente entre las universidades privadas y públicas, 10 de los cuales corresponden a hombres y 22 a mujeres. El número de entrevistados obedece al punto de saturación discursiva. El acceso a los sujetos se efectuó a través de la identificación de informantes clave, técnica de bola de nieve y listados confeccionados por los docentes-directivos. En cuanto a los criterios de inclusión de los sujetos diremos que nos interesó el que hubiesen superado el primer año de su formación, que fuesen estudiantes matriculados al momento de realizar las entrevistas, y que estuviesen dispuestos a compartir sus experiencias en contexto de entrevista.

El análisis de los discursos lo efectuamos de la forma que a continuación se detalla. En primer lugar realizamos una lectura de las entrevistas de forma individual separándolas según tipo de universidad (pública/privada); en segundo lugar las integramos y comenzamos un proceso de codificación abierto, sin perder de vista algunas categorías apriorísticas tales como concepto de educación y rol docente. Por último, realizamos un análisis integrado de los discursos con el fin de construir categorías más generales y así poder establecer relaciones entre las mismas; procedimiento que en su conjunto se sustentó en el paradigma de la codificación propuesto en la teoría fundamentada.

\section{Presentación de resultados}

\subsection{Acerca del discurso institucional y su influencia en las imágenes de los estudiantes de magisterio de primaria}

En el contexto del Plan Bolonia y el Espacio Europeo de Educación Superior (EEES), las instituciones de educación universitaria españolas han tenido que diseñar e implementar un conjunto de modificaciones en sus programas de formación, la más importante de las cuales corresponde a un ajuste curricular hacia un modelo por competencias que se conjuga con el paradigma de educación a lo largo de la vida. Las competencias posibilitan vislumbrar que, a través de los planes de estudios, se modela un tipo de acción educativa para la formación del profesorado que se dirige fundamentalmente al desempeño profesional en el aula. Lo cual, desde nuestro punto de vista, constituye otro de los factores que explican que los estudiantes del Grado de Magisterio (/Maestro) de Educación Primaria "naturalicen" los contenidos educativos, en el sentido expuesto en páginas precedentes. A los estudiantes no se les enseñan contenidos (sean de geografía e historia, sean de matemáticas, sean cuales sean) sino cómo enseñar contenidos, que es lo que se les presenta como (y va a constituir) sus señas de identidad profesional. Ello, no cabe duda, es una 
obviedad en el ámbito del magisterio, pero ya no son tan obvias sus consecuencias: entre ellas, esa naturalización de los contenidos que ahora estamos tratando, puesto que esta situación, en que no se reflexiona sobre ellos, al no ser - por así decirloobjeto de interés en la formación inicial del profesorado, favorece que sean tratados "como dados" o, al menos, no contribuye a que se les problematice.

En el marco de las reformas que se han derivado de la inclusión de la Universidad española en el EEES, los estudios relacionados con la formación inicial del profesorado, además del cambio de orientación teórica que ha llevado a establecer unos currícula basados en competencias, también han experimentado otros cambios. Uno de los más evidentes consiste en haber adquirido la categoría de grado universitario, dejando de ser una diplomatura. Lo cual, a su vez, ha incidido en dos aspectos principales de estos estudios: por una parte, en el aumento del tiempo dedicado a las prácticas externas; por otra parte, en las estrategias pedagógicas desarrolladas por los docentes, que ahora tienden a priorizar, sobre todo, la realización por parte de los alumnos de trabajos en equipo en las diferentes materias que conforman el plan de estudios.

El incremento de prácticas externas y los cambios en las estrategias docentes están relacionados con el postulado de aprender haciendo, y el énfasis que se pone en el trabajo personal del alumno lo está con el aprender a aprender; ambos permeados por el aprendizaje a lo largo de la vida tan ligado a la pedagogía contemporánea. Con ello se ha tratado de responder a las exigencias del EEES y del Plan Bolonia y, paralelamente, a las de una formación por competencias. Estos elementos vuelven a evidenciar, en su conjunto, un discurso hegemónico en la formación inicial del profesorado que se sustenta en los postulados del paradigma del aprendizaje basado en competencias, en cuya conformación ha tenido un peso importante el enfoque constructivista.

Un aspecto fundamental en el que se ve reflejado este discurso hegemónico es la relevancia que los estudiantes otorgan a las prácticas externas, pues consideran que es la mejor forma de aprender. De hecho, los alumnos entrevistados a menudo aseguran que, en las prácticas realizadas en los centros escolares, han aprendido más que en las horas de clases presenciales que han tenido a lo largo de su carrera. Una muestra de ello se encuentra en el discurso de Adam.

Muchísima gente que ha terminado la carrera me lo ha dicho: que lo único que vale son las prácticas, por lo menos en esta Universidad. (Adam. 20-25 años. Estudiante Universidad Pública)

De hecho, pone en evidencia la necesidad, sentida como tal por los estudiantes de magisterio, de articular adecuadamente los saberes conceptuales con la puesta en práctica de dichos saberes con el fin de que tengan "utilidad para la vida real".

Muchas asignaturas no tienen su utilidad. Didácticas estúpidas: algunas que ni se aplican, otras son teorías de las didácticas pero que tienen que darse en didáctica y que, en realidad, son muchas cosas que no vas a aplicar con los niños. Pues, un montón, un montón. No sé si te lo habrán dicho más estudiantes, pero yo con los de 
mi clase opinamos lo mismo: que no tiene utilidad, nada [...]. Muy poquitas asignaturas tienen utilidad en la vida real. (Adam. 20-25 años. Estudiante Universidad Pública)

Lo que se desprende del discurso de Adam, como de los discursos de otros alumnos entrevistados, es la idea de que el maestro se construye a través de la práctica. Idea que podría estar poniendo asimismo de manifiesto que la pérdida del monopolio sobre la información que ha experimentado el profesorado tanto de primaria y como de secundaria (pérdida a la que se refieren autores como Martín y Coll, 2003) no es privativa de él, sino que también se extiende al profesorado universitario. Así, los contenidos teórico-conceptuales pueden ser trabajados, de manera autónoma (véase el discurso de Concepción un poco más abajo), a través de la revisión de literatura y de documentos especializados fácilmente accesibles hoy en día, por lo que los conocimientos del profesor (y, en consecuencia, las asignaturas donde se transmiten) son relativamente prescindibles. Sin embargo, el saber práctico se obtiene haciendo, es decir, practicando in situ, lo que remarca aun más - a ojos de los estudiantes- el valor de las prácticas externas. Concepción nos pone un ejemplo referido a la enseñanza del inglés:

En inglés vemos la historia de los métodos para enseñar inglés a personas hispanohablantes. Discúlpame, pero ¿de qué me sirve a mí conocer la historia de los métodos que se han utilizado? Lo que me interesa es saber cuál es el método que mejor funciona. Quiero saber cuál es el que funciona, cuál el que no y saber de qué forma enseñar mejor. Yo quiero enseñarle inglés al niño, no decirle: "Es que hace muchos años los ingleses...". Es que no. Lo que me importa es enseñar y saber cuál es el que debo aplicar. El tema tiene que ver con la utilidad de las cosas. (Concepción. 20-25 años. Estudiante Universidad Privada)

Talía también argumenta la importancia del saber práctico pero desde otra perspectiva, puesto que no lo enfoca en términos de un saber hacer, sino de un saber ser.

Hay una cosa buena en esta universidad y es que tienes prácticas desde primero [...]. Al menos te sirve para ponerte en situación y darte cuenta de lo que viene,

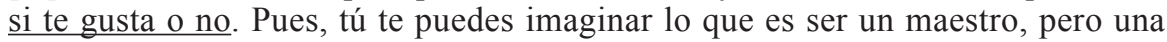
cosa distinta es estar ahí siendo maestro. (Talía. 30-35 años. Estudiante Universidad Privada)

Lo expuesto hasta ahora deja claro el valor que los estudiantes de magisterio dan, dentro del proceso de su formación inicial docente, al saber práctico (entendido como un saber hacer o como un saber ser/estar y, por tanto, como un saber útil) y a las prácticas para adquirirlo (a las prácticas externas pero también a las que cabría calificar de internas, en el sentido de realizadas en las aulas universitarias); lo que expresa, a la par, la demanda de una formación eminentemente instrumental. La obtención de herramientas dirigidas a conformar ese saber hacer como maestros se torna central para estos estudiantes, en desmedro de los conocimientos que califican de teóricos, que son ampliamente criticados por ellos. Autores como Marchesi 
(2000), afirman que los contenidos teórico-conceptuales, adecuadamente articulados con la práctica, otorgan posibilidades de reflexión y análisis crítico que contribuyen a una formación más integral de los futuros maestros de primaria. Sin embargo, como se ha visto, los estudiantes de magisterio plantean la teoría y la práctica en términos dicotómicos, lo cual es posible que se deba, al menos en cuanto a la manera en que se presenta en sus discursos, a que mezclan sin solución de continuidad elementos curriculares diferentes, atribuyendo la cualidad de práctico o de teórico indiscriminadamente a los contenidos de la enseñanza-aprendizaje, a los saberes adquiridos o que se busca adquirir (a las competencias profesionales, por tanto) o, por último, a los modos o las metodologías para adquirirlos. Todo ello invita a pensar que el poco peso que los futuros profesores asignan a lo teórico, incluidos los contenidos teórico-conceptuales, puede llevar a la conformación de una escasa capacidad para (y de un escaso interés por) la reflexión crítica sobre el quehacer educativo en la escuela, y más concretamente sobre qué deberían (o no) enseñar y en qué momentos de la vida de los aprendices.

Aunque a nivel programático se declare que se quiere formar a profesionales reflexivos, se puede apreciar que los currícula de los diferentes grados de magisterio de educación primaria (al menos, los de las universidades que han constituido nuestro universo de investigación) direccionan esa reflexión hacia la gestión de (/ hacia la acción pedagógica en) el aula. Es lo que ocurre, por ejemplo, en una de las universidades públicas estudiadas, en cuyo currículo se asocian, aproximadamente, el $50 \%$ de los créditos obligatorios a asignaturas cuyos nombres incluyen la palabra didáctica. En cuanto a los programas de las asignaturas básicas en las que se abordan temáticas vinculadas con la psicología del desarrollo y de la educación, la sociología de la educación y/o la teoría e historia de la educación, se observa lo siguiente: 1) una clara presencia del constructivismo pedagógico, 2) la concesión de una escasa importancia, en términos de tiempo que se le dedica, a cuestiones relativas a la vinculación de la escuela con la comunidad y/o con la familia; y 3) una menguada referencia a planteamientos críticos (/literatura crítica) en las asignaturas ligadas a la sociología de la educación. Lo que, en su conjunto, vuelve a confirmar la idea de que, en la formación inicial del profesorado, la labor docente se orienta a realizar una lectura de la realidad que se restringe al espacio interno del aula y, como mucho, de la escuela, a priorizar el cómo enseñar sobre el qué y el cuándo enseñar, y a prestar una atención especial a la gestión del aula para así conseguir unos objetivos educativos que han sido trazados por las administraciones escolares sobre la base de los principios, recomendaciones y ordenanzas de organismos supranacionales.

\subsection{Por qué el énfasis en el cómo: una aproximación desde el discurso de los estudiantes}

En las elaboraciones discursivas de los estudiantes de magisterio se pone de manifiesto el protagonismo que otorgan a los aspectos técnico-pedagógicos para su formación como maestros, y hacen hincapié - como ocurre también con los planes de estudios - en lo didáctico y en las técnicas pedagógicas que les faciliten el proceso de concreción del currículo a nivel del aula. El énfasis en estos aspectos 
técnico-pedagógicos evidencia una visión naturalizada de los contenidos educativos establecidos a nivel curricular, es decir, un no-cuestionamiento del qué, por qué o cuándo enseñarlos, erigiéndose el cómo enseñar en el eje de las inquietudes de los estudiantes de magisterio.

Así, a pesar de que, según Marchesi y Martín (1998), los profesores han ido asumiendo nuevas responsabilidades desde que se implementó la LOGSE en España (en cuanto a decisiones relativas al curriculum, la oferta educativa y la gestión de los recursos, por ejemplo), cuando se analiza el discurso de los estudiantes de magisterio de primaria - más de una década después - , se constata que su foco de interés se sitúa en la organización de la enseñanza y se concreta en la adquisición de técnicas y estrategias para la misma. Con relación a ello, otro espacio donde se aprecia esta inclinación hacia cómo enseñar se encuentra en las expectativas que tenían al ingresar en la carrera de magisterio de primaria. Un claro ejemplo de ello se halla en el siguiente fragmento de la entrevista realizada a Guadalupe.

Yo supongo que tenía la idea de que me enseñarían un poco más a cómo tratar a los niños, cómo enseñarles mates, lengua, métodos. No tanto cosas que te lo estudias para el examen, te lo estudias y ya a las dos horas no te sirve para nada (Guadalupe. 20-25 años. Estudiante Universidad Pública).

Expectativas como las de Guadalupe tienen su origen en las representaciones que los estudiantes manejan acerca del rol de maestro, que - por lo que se desprende de las entrevistas - traen ya consigo, al menos en la cuestión ahora considerada, cuando ingresan al Grado de Magisterio (/Maestro) en Educación Primaria: el maestro es quien enseña un conjunto de conocimientos que vienen definidos para las diferentes áreas curriculares, por lo que es de esperar que, durante el proceso de formación inicial docente, se proporcionen herramientas para cumplir con ese rol de forma adecuada.

Como vimos en las páginas precedentes, uno de los motivos que orientó, en los años 80 ' sobre todo, la discusión en torno a la necesidad de reformar el sistema escolar español tenía relación con las tasas de abandono y la desmotivación existentes entre los alumnos. Esta última fue percibida entonces como una de las causas principales que habían dificultado el quehacer docente y, consecuentemente, incrementado el fracaso escolar (Marchesi, 2000). Se trata de una preocupación que es posible reconocer aún hoy en los discursos de estudiantes de magisterio de educación primaria. De hecho, en el marco de estos discursos, la inquietud acerca de cómo enseñar se suele asociar a la necesidad de motivar, lo que sugiere que estos estudiantes hacen depender la motivación de las formas que tiene el docente de trabajar con los alumnos/as. Qué estrategias pedagógicas seguir y/o qué actividades programar son preguntas que reflejan una preocupación por los aspectos técnico-pedagógicos (no por los político-ideológicos) de los procesos de enseñanza-aprendizaje; una preocupación que se encuentra, por ejemplo, en las palabras de Mónica:

He tratado de explicar en una clase lo que significa la familia en inglés, y había una niña marroquí que es que no... es como si no cayera, hasta que salió a la pizarra y 
le expliqué los nombres de mi familia y luego hice que escribiera los nombre de su familia. Y me llamó la atención muchísimo porque participó muchísimo. Entonces, ahí está la labor del profesor. El contenido está ahí para el alumno y el alumno puede acceder a él solito, de distintas maneras. Lo que el profesor tiene que hacer es facilitar el canal para que el contenido le llegue al alumno y para que le interese. Si a un alumno no le entra por aquí, tal vez le entra de otra manera, o le llega de otra manera, y eso es lo que tenemos que hacer como profesores. (Mónica, 30-35 años. Estudiante Universidad Privada)

De las palabras de Mónica, se desprende una cuestión importante relativa a la imagen que los estudiantes de magisterio poseen de su futuro rol como maestros. Esta consiste en actuar/hablar/pensar como si los contenidos educativos "estuvieran ahí", pre-definidos, y que, por tanto, el maestro lo que debe hacer es "facilitar el canal para que esos contenidos les lleguen a los alumnos", puesto que se piensa que, al contrario de lo que ocurría antes, en la actualidad son fácilmente accesibles para todos. Ahora bien, el discurso de Mónica es, en cierto sentido, muy poco constructivista, puesto que el rol que atribuye al maestro no deja de tener relación con una concepción tradicional de la enseñanza, pues sigue asociándola a la transmisión de contenidos (no a la construcción de conocimientos), sólo que ahora el cometido del maestro no consiste en transmitir dichos contenidos, sino en favorecer su transmisión (en ese "facilitar el canal para que les lleguen a los alumnos", que recordábamos más arriba). De modo que los contenidos son tratados como objetos reificados (Díaz de Rada y Velasco, 1996), poniendo de manifiesto cómo el constructivismo es interpretado - tanto por alumnos como por docentes que intervienen en la formación inicial del profesorado- desde esquemas viejos, como es el clásico de entender la educación como transmisión-adquisición.

Otro aspecto que se desprende del discurso de Mónica es que el maestro debe ser un agente motivador, ha de contribuir a la generación de un clima de aprendizaje adecuado, atendiendo a la vez al contexto y a la diversidad del alumnado presente en el aula. En este escenario, en el que el maestro debe motivar para enseñar, el profesorado debe ser un guía. Estos elementos discursivos han permeado los espacios institucionalizados en los que se realiza la formación inicial del profesorado. Pero como se desprende del discurso de Mónica, han sido entendidos de manera muy sui generis, y así han sido transmitidos a los futuros titulados en maestro de educación primaria que hemos entrevistado.

En cuanto al papel de guía que ha de asumir el profesor en su praxis pedagógica, ha entrado claramente a formar parte (al menos en términos declarativos) del discurso de los estudiantes de magisterio de primaria, y así, por ejemplo, Camila expone de forma enfática la importancia que tiene orientar a los alumnos durante el proceso de enseñanza y aprendizaje:

Como decía, tenemos que guiar. No tenemos sólo que dar contenidos que después se olvidan, sino que tenemos que ayudar a que ese niño, cuando termine, sea capaz de continuar él solo. Pero hay que ayudarles a razonar sobre esas cosas abstractas, intangibles. (Camila. 25-30 años. Estudiante Universidad Pública) 
Este papel de guía es, desde el punto de vista de estos estudiantes, el que precisamente permitirá transferir protagonismo al alumnado, pues ésta es condición sine qua non para lograr los tan ansiados aprendizajes significativos.

El estudiante tiene que ser más protagonista, pues el profesor tiene que ser una referencia, pero que estimula, no uno que dicta y que no sabe salirse de la estructura (Francisca. 20-25 años. Estudiante Universidad Pública).

Tanto en las entrevistas hechas a estudiantes como en los informes de prácticas, se aprecia una cierta insistencia en la idea de que el profesor es un guía, lo que quizá pueda entenderse si se tiene en cuenta el entramado discursivo mayor que opera a nivel institucional en torno a la profesionalización del maestro.

De ser transmisores de información, debemos convertirnos en apoyo continuo y cercano al desarrollo del alumno, (en) conductores del proceso de construcción del aprendizaje, preocupados por investigar, una vez y otra también, cómo aprende y cómo no, por dónde se pierde (Sandra. Estudiante Universidad Privada. Informe de Prácticas, pp.34).

Aunque la función de "guía en el proceso de búsqueda" es reconocida en el discurso de los estudiantes de magisterio de primaria como uno de los roles del maestro, nada hace pensar que éste se cumpla efectivamente cuando trabajen en las aulas, puesto que, a pesar del conjunto de reformas que en materia escolar se han realizado en España, las lógicas burocráticas y las inercias de la escuela permanecen. Tanto es así, que los contenidos curriculares continúan siendo enseñados de manera abstracta y descontextualizada, es decir, sin vincularse con las experiencias de los alumnos, aunque muchas veces se declare lo contrario. De modo que el énfasis no está puesto en la educación para la vida, sino más bien en los conocimientos técnico-científicos, tratados además como verdades establecidas en lugar de como procesos de identificación / descripción / interpretación / explicación de la realidad.

Podríamos decir que uno de los elementos que llevan a comprender por qué los contenidos escolares son naturalizados (tratados como algo que está ahí, que no se problematiza ni se cuestiona su pertinencia), a la par que se enfatiza el cómo ense$\tilde{n} a r$ hasta el punto de fetichizarse a veces, es que los estudiantes no han desarrollado una práctica docente con suficiente continuidad como para lograr elaborar procesos reflexivos derivados de su quehacer docente, sino que sus reflexiones se generan más bien en el marco institucionalizado de la formación inicial y, por tanto, respondiendo a exigencias formales orientadas a conseguir la credencial que los faculte para trabajar como maestros de primaria.

En síntesis, a pesar de que, en la formación inicial del profesorado, se plantea el modelo constructivista como fundamento teórico de la futura acción educativa, los estudiantes de magisterio de primaria restringen su rol como maestros al plano técnico, circunscribiendo su participación en el proceso de enseñanza-aprendizaje a cómo enseñar, de modo que dicho rol queda más próximo al de un sujeto que transmite conocimientos preestablecidos (o facilita canales para su transmisión — como 
se ha visto más atrás-) que al de un sujeto que contribuye a que los alumnos los construyan. ¿Por qué? Tal vez porque no han tenido aún la posibilidad de participar sostenidamente en equipos docentes así como tampoco de poner en práctica una acción educativa a nivel de aula, por lo que no han podido desarrollar los procesos esenciales que los modelen como profesionales reflexivos; o también por el hecho de que los nuevos paradigmas (como el constructivismo pedagógico) se construyen desde esquemas 'viejos', ideas previas o preconceptos que no son sometidos a crítica (como exigiría - paradójicamente- el constructivismo pedagógico); o bien, por esa naturalización de los contenidos de la que hablaba antes; o porque el entorno burocrático en que deben desarrollar su labor no es muy propicio a poner en práctica las innovaciones que, en cambio, pregonan o declaran como deseables.

\section{A modo de conclusión. La práctica y lo práctico en la enseñanza: una síntesis como consecuencia de la tecnología instruccional}

Creemos adecuado considerar que los valores sociales que predominan en una sociedad inciden de forma directa en la conformación de los diversos roles que deberá desempeñar el profesor. Si es verdad que los valores predominantes en las sociedades occidentales son el individualismo y el pragmatismo, como plantea por ejemplo Bajoit (2003), es de esperar que estén presentes en los profesores y —para el caso específico de este estudio- en los futuros maestros de primaria en el momento de su desarrollo profesional.

El predominio de estos valores en la sociedad tendría pues un importante peso cuando se trata de comprender/explicar las transformación del rol de maestro, así como el hecho de que la relevancia que antaño se otorgaba a la vocación haya sido superada con creces hoy en día por la inclinación hacia el pragmatismo (Dubet, 2008). En este contexto, Giroux (1990) afirma que es necesario reflexionar sobre los procesos educativos formales que tienen lugar en diferentes ámbitos, es decir, no sólo en la educación primaria, sino también en la formación inicial y en la formación continua del profesorado, en los cuales, desde su punto de vista, se consolida una relación inversamente proporcional entre la tecnocracia educativa y la pedagogía crítica radical. Por un lado, según asegura Giroux (1990), se observa la supremacía del discurso gerencial y administrativo centrado en la eficacia y el control; por el otro, un retroceso o pérdida de interés por el desarrollo de una alfabetización crítica y una ciudadanía militante. Concordamos con este autor respecto a la importancia de concebir al profesor como intelectual transformativo, en tanto que agente social que contribuye a la producción de cultura por medio del análisis crítico, el diálogo y la acción pedagógica negociada con los alumnos, sus familias y la comunidad en su conjunto. Asimismo, vemos con preocupación - al igual que el autor-que esta posibilidad se desvanece y dificulta en el escenario actual. Esta tendencia, de darse tal como la presenta Giroux (1990), traería consigo repercusiones importantes en la imagen que los futuros maestros de primaria se forjan acerca de su quehacer profesional, puesto que la idea de intelectual transformativo a la que este autor apela no tendría cabida cuando lo que importa es la eficacia y la eficiencia de la acción pedagógica. Se construye una imagen del quehacer profesional en la cual adquieren 
centralidad los aspectos técnicos que cobran cuerpo ya en el proceso de formación inicial del profesorado y que, de acuerdo a autores como Coba (2011), Egido (2011) y García (2009), tienden a perpetuarse durante el desarrollo de la carrera docente.

Volvamos a los espacios escolarizados. La fuerza de la tecnocracia, el predominio del discurso técnico en la formación inicial docente, la marginación de los aspectos morales y éticos en el proceso de enseñanza-aprendizaje que se producen no sólo en la escuela primaria (o secundaria o universitaria) sino ya desde los procesos de formación inicial del profesorado, ponen en evidencia la configuración de discursos y prácticas institucionalizadas que expresan y actúan sobre la base de una forma particular de entender la educación: como transmisión de conocimientos, sobre todo técnicos, que se orientan a contribuir a la completud de un sujeto que asume, sin cuestionarlo, el funcionamiento de la sociedad, pues todo hace pensar que las críticas que tienen lugar en los espacios escolares están enfocadas principalmente a aquellos aspectos que, a nivel macro y/o microsocial, entorpecen el normal modo de funcionar del mundo.

En la formación inicial del profesorado, de aquellas universidades que conformaron nuestro universo de investigación, prima la idea de una educación neutral a nivel político, comprometida principalmente con los aspectos técnico-pedagógicos, es decir, con aquellos aspectos que orientan las imágenes que los estudiantes del grado de primaria, tienen con respecto al rol que han de desempeñar en las escuelas. Son estos conocimientos técnicos los que - de acuerdo a las representaciones que manejan- otorgan el status profesional al profesorado. De este modo, la dimensión político-ideológica, tan relevante para la acción pedagógica transformativa, al estar ausente de los procesos de formación inicial del profesorado, ve dificultado aun más su desarrollo en el ejercicio profesional de estos futuros maestros, especialmente en los inicios del mismo. Puede entenderse así por qué resulta más cómodo a los estudiantes de magisterio de primaria centrarse en asumir un currículo preestablecido por la administración educativa, pues 1) les orienta acerca del proceso de enseñanza que han de desarrollar con el alumnado, teniendo una base normativa que respalda su proceder frente a otros docentes de mayor experiencia; 2) les proporciona una base conceptual para la elaboración de un discurso técnico especializado que a su vez les permite - en caso de considerarlo necesario - el distanciamiento con respecto a las familias apoyando sus argumentos en su condición de sujeto experto; y 3) les provee de una batería conceptual y técnica que facilita una aparente objetivación de las formas de evaluar los aprendizajes, lo que opera como resguardo frente a potenciales conflictos con los alumnos/as y sus familias. En virtud de lo expuesto, puede plantearse que lo que prima en el contexto de la formación inicial del profesorado y, muy probablemente durante los primeros años de la carrera docente, es una suerte de fetichización de los dispositivos técnicos cuyo reflejo más claro se encuentra en la hegemonía de una tecnología instruccional dentro de los espacios escolares, sean del nivel que sean. 


\section{Referencias bibliográficas}

BAJOIT, Guy.

2003 Todo cambia: análisis sociológico del cambio social y cultural en la sociedad contemporánea. Santiago: LOM.

COBA, Eduardo.

2011 "La formación inicial del profesorado en el Espacio Europeo de Educación Superior". CEE Participación Educativa. 16: 31-38.

DÍAZ DE RADA, Ángel; VELASCO, Honorio.

1996 "La cultura como objeto". Teoría y Práctica de la Educación, 17: 6-12.

DUBET, François.

2008 "El declive y las mutaciones de la institución”. En Jociles, M. y Franzé, A (Eds.). ¿Es la escuela el problema? Perspectivas socio-antropológicas de etnografía y educación. Madrid: Trotta, 217-246.

EGIDO, Inmaculada.

2011 "Cambios y dilemas en la formación del profesorado (1961-2011). Cincuenta años de historia de España en perspectiva europea”. Tendencias Educativas, 18: 33-50.

GARCÍA, María.

2009 "Formalidad e informalidad en el proceso de aprender a enseñar". Revista de Educación, 350: 31-56.

GIROUX, Henry.

1990 Los profesores como intelectuales. Hacia una pedagogía crítica del aprendizaje. Barcelona: Paidós.

MARCHESI, Alvaro.

2000 Controversias en la educación española. Madrid: Alianza.

MARCHESI, Alvaro; MARTÍN, Elena.

1998 Calidad de la enseñanza en tiempos de cambios. Madrid: Alianza.

MARTÍN, Elena; COLL, César.

2003 Aprender contenidos, desarrollar capacidades. Intenciones educativas y planificación de la enseñanza. Barcelona: Edebé.

POZO, José.

1996 Aprendices y maestros. Madrid: Alianza.

VARELA, Julia.

2007 Las reformas educativas a debate (1982-2006). Madrid: Morata. 